average, this represents 1.61 of water intake per day for females and 21 of water intake per day for males. French national dietary survey (INCA 2) included more than 4000 people aged 3-79 years between 2006 and 2007 . Volunteers declared all their food and fluid intake during a 7-d period. Main results of the present study showed (i) total consumption of fluids are 745, 856, 943, 1022, 1491, 1626 and $1422 \mathrm{ml}$ for 3-6, 7-10, 11-14, 15-17, 18-34, 35-59 and $\geq 60$-year-old population, respectively; (ii) $80 \%$ of French adults, 18-79 years of age, drink less than 21 of fluids daily; (iii) water is the most consumed fluid in France $(\sim 800 \mathrm{ml}$ on average); (iv) the $25 \%$ of higher sugared beverages adult drinkers consume more than $450 \mathrm{ml}$ of SB per day; (v) SB represent $20 \%$ of total fluid consumption for children and adolescents (3-17-year-old); and (vi) SB represent on average $17 \%$ of simple sugar daily intake for children and adolescents. A very large part of French people should drink more in order to better satisfy water adequate intake proposed by EFSA. In addition, even if water remains the most consumed fluid in France, the consumption of sugared beverages represent significant intake of sugar and simple sugar, especially for children and adolescents.

Poster Abstracts: Metabolic and Genetic Aspects 22 - Tracking changes in metabolic function with changes
in body composition G O'Malley ${ }^{1,2}, V^{\prime}$ Northrup $^{2}$ and S Caprio'

${ }^{1}$ Department of Paediatrics, School of Medicine, Yale University, New Haven, CT, USA: ${ }^{2}$ The Physiotherapy Department, The Children's University Hospital, Dublin, Ireland

Introduction: It is unknown whether changes in body composition measured by either DXA or BIA are predictive of changes in metabolic health in obese children.

Method: Children completed a DXA scan, BIA measurement, an oral glucose tolerance test and a hyperglycaemic clamp at baseline ( $n$ 113) and follow-up ( $n$ 64). Differences between sample characteristics and metabolic outcomes were compared, as were changes over time. Linear regressions were used to test the association between change in body composition variables and change in metabolic outcomes adjusting for baseline age, BMI $Z$-score, ethnicity and gender.

Results: For every $1 \%$ increase in PF over time, there was a decline in $\mathrm{M}$ and $\mathrm{M} / \mathrm{LBM}$ of 0.13 and 0.17 , respectively, using BIA. Each kilogram increase in BiaFM was predictive of a 0.08 decline in $\mathrm{M}$, a $0 \cdot 13$ decline in $\mathrm{M}$ / LBM, a 1.49 decline in IGI and a 4.31 decline in DI over time $(P<0 \cdot 05)$. Each kilogram increase in DXFM was predictive of a 0.09 decline in $\mathrm{M}$, a 0.02 decline in $\mathrm{M}$ / LBM, a 1.68 decline in IGI and a 4.79 decline in DI over time $(P<0 \cdot 05)$. For each $1 \mathrm{~kg}$ increase in FFM, declines in M/LBM, IGI and DI were observed $(0 \cdot 19 ; 1.94$ and 5.66 for DXAFFM and $0 \cdot 21 ; 3 \cdot 37$ and $9 \cdot 71$ for BiaFFM).

Conclusions: Changes in PF, FM and FFM over time as measured by DXA and BIA can indicate associated change in metabolic health in obese children and adolescents. BIA could be used easily in a clinical setting to track such metabolic changes. DXA - dual energy X-ray absorptiometry; BIA - bioelectric impedance analysis; $\mathrm{PF}$ - percent fat; $\mathrm{M}$ - insulin sensitivity; $\mathrm{M} / \mathrm{LBM}$ - insulin sensitivity/lean body mass; IGI - insulinogenic index; DI - disposition index.

Funding: Research related to this work was made possible due to grants provided by NICHD (R01HD40787) and The Irish Fulbright Commission.

\title{
23 - Serum neopterin and tryptophan concentrations in obese children
}

D Weghuber ${ }^{1,2}$, D Fuchs ${ }^{3}$, V Krosslhuber ${ }^{1}, \mathrm{H} \mathrm{Mangge}^{4,2}$ and D Zaknun ${ }^{5}$

${ }^{1}$ Department of Pediatrics, Paracelsus Private Medical School Salzburg, Salzburg, Austria: ${ }^{2}$ Obesity Academy Austria, Austria: ${ }^{3}$ Division of Biological Chemistry, Biocenter, Innsbruck Medical University, Innsbruck, Austria: ${ }^{4}$ Clinical Institute of Medical and Chemical Laboratory Diagnostics, Medical University of Graz, Graz, Austria: ${ }^{5}$ Department of Pediatrics, Medical University of Vienna, Vienna, Austria 